\title{
Essays
}

\section{Obstacles to setting up and improving a journal in today's scientific community in Iran}

\author{
Kianoush Khosravi-Darani \\ Research Department of Food Technology, National Nutrition and Food Technology Research Institute, Faculty of \\ Nutrition and Food Sciences, Shahid Beheshti University of Medical Sciences, Tehran, Iran; k.khosravi@sbmu.ac.ir; \\ ORCID: /0000-0002-0269-6385
}

DOI:10.20316/ESE.2019.45.18006

\begin{abstract}
Setting up and improving a scientific journal contributes greatly to promoting science in all societies. However, in many countries, and especially in the developing countries, editors of scientific journals find it difficult to do so because they lack appropriate training in establishing and running a journal. All such problems and challenges are reviewed, including those involved in ensuring that the journal is covered by major indexing services and databases. The editor of a scientific journal often has to balance conflicting needs and streamline the everyday operation of the journal while maintaining the integrity of its scientific content. The editorial board, especially the chief editor, is under pressure from different players that make up the publication chain. Practical experience gained during setting up a new journal is described along with some suggestions on improving publications quality and coverage by indexing services.
\end{abstract}

\section{Keywords}

Journal publishing, indexing of journals, peer review, revising research papers, scientific journals, scientometrics.

\section{Introduction}

The publication chain of a scientific journal is complex and involves players with different skills, with the journal's editor playing the important role of coordinating and balancing the different links and keeping everyone happy (authors, reviewers, readers, staff, members of the editorial board, the publisher, and indexing services). The task is even more challenging when a new journal is lanuched, especially in such developing countries as Iran. Ethical issues are a major concern, as is the need to improve the quality of the journal and ensuring that it is indexed by major databases (which may change over time). ${ }^{1}$ The editor often has to balance conflicting needs and streamline the everyday operation of the journal while maintaining the integrity of its scientific content.

In writing on this topic, we not only reviewed the relevant literature but also spoke to more than twenty experienced editors and other stakeholders, reviewers, and government officials actively involved in running scientific journals and familiar with the problems of scholarly publishing. Our discussions focused on the difficulties they faced and on how they surmounted those difficulties. The following account deals with different links in the publishing chain and other related issues.

\section{Negligent authors}

Many of the problems faced by journal editors are due to the careless approach of authors. Such problems include the following: incorrect references, failure to sign the covering letter by all the authors, missing photos and other types of illustrations, withdrawing accepted manuscripts at the last minute, need for repeated reminders, not addressing all the queries raised by the referees or not separating the response from the revised manuscript (by sending a single file instead of two separate files), inability to mark proofs (sent as a file in PDF) using the standard proofreader's marks, repeated requests related to changing the order in which the names of authors are listed or even adding or removing the names, and pressing for early publication without any payment. ${ }^{1-4}$

\section{Lack of a culture of research}

Although a quarter of all the scientists in the world live in the developing countries, their contribution to science is less than $2 \% .^{3}$ The fact that publication was not recognized as a form of merit in medical schools until recently meant that medical researchers had no incentive to publish. The scientific methodology is now being taught much earlier in the curriculum to foster the development of scientific communities. Another factor that weakens the research culture in Iran is lack of appreciation of research as a worthwhile career and the consequent lack of respect and status. Also, research was not considered a sole career for scientists so far; however, things are changing now. ${ }^{3}$

Among the more specific problems are lack of novelty in most studies, bias in sample selection and even in peer review, and lack of systems for data sharing leading to waste of time and energy as each researcher reinvented the wheel as it were.

\section{Inadequate peer review}

The process of peer review is poorly understood, which can also result in controversies. Godlee, the editor of BMJ, remarked, "Peer review is expensive, slow, subjective, biased, open to abuse, patchy at detecting important methodological defects and almost useless at detecting fraud and misconduct". 5 Perhaps peer review is not a reliable basis for rejecting or accepting submissions, but it is the best instrument we have at the moment.

Lack of competent and qualified reviewers may make small journals more lenient in accepting submissions and employjng poorly qualified reviewers. ${ }^{3}$ 
Recognizing the contribution of peer reviewers publicly is better than offering monetary rewards in motivating them. Good reviewers should be available, responsible, and expert in their respective fields. Making a formal contract with good reviewers helps in eliciting faster and more detailed responses from them. If a reviewer often fails to spot major errors, makes faulty judgements, or delays in returning the assigned manuscripts, the contract should be cancelled. Sometimes, post-publication reviews can speed up the publication process. ${ }^{3}$ Predatory or deceptive journals are open access and usually dispense with peer review and standard editorial processes but charge a publication fee from authors. ${ }^{6}$

Researchers have many resources to learn more about peer review. These resources include practical and structured training courses, online guidelines and videos, webinars, journal clubs to share post-publication reviews, critical appraisal meetings to conduct pre-publication reviews, and experiences of the members of editorial boards, research supervisors, and mentors. Such resources meet the needs of the academic community and help in ensuring better peer reviews and guiding early-career researchers on conducting peer reviews and journal editors in finding competent reviewers. ${ }^{7}$

A team of Croatioan editors developed a game, the peer review card exchange game, and used it for teaching integrity and ethics as part of a training programme on peer reviewing. The results were positive, and the trainee editors said that the game helped them to become responsive, competent, impartial, discrete, constructively critical, and responsible. ${ }^{8}$

\section{Conflicting local regulations}

Science ministries in many countries encourage university faculty and other members of the academic and research community to publish in those journals that are indexed by the more reputable and prestigious databases but offer only inadequate financial support to universities and research institutions: limited budgets lead to limited research, and papers based on such research are often rejected by those very journals in which the government expects its researchers to publish. In Iran, besides the Ministry of Health, which covers medical journals, the Ministry of Science, Research, and Technology approves and monitors many journals in other domains. These journals are bound by specific laws and regulations of their respective ministry, and editors of journals covered by both the ministries find it difficult to reconcile the differing requirements and to fulfil administrative chores.

The large number of journals leads to such problems as low-quality papers and insufficient supply of good-quality papers to any newly established journal. Of the 403 medical journals in Iran, 242 are in English, and their first obligation is to be indexed in PubMed Central (PMC), Scopus, and the Web of Science. In Iran, the scientific community also faces other serious problems that inhibit genuine research. One major problem is that academics and researchers are evaluated and ranked every year based on the number of papers published and the number of citations received in the previous year. Linking funding and promotions to such metrics pushes every smart researcher to prefer quantity to quality and originality.

\section{Poor support from editorial boards}

Most of the editors from the developing world have had no specific training in editing and publishing; they have acquired the skills through experience on the job. Despite their efforts, the task offers no financial rewards but only professional satisfaction, although making decisions at the policymakers level and having an impact on science publishing bring their own reward. However, those editors and members of editorial boards who are not satisfied with these intangible rewards put in little effort in their job, and the journals they edit suffer from that passive stance. Sometimes, it is useful to engage experts as section editors or guest editors: this approach can increase the speed and quality of revision. Yet, it is difficult to find competent editors with the necessary expertise with good standing who can network with other experts and enlist them as reviewers and are willing to shoulder the responsibility of responding promptly to all emails and scrutinizing referee reports and the authors' responses to those reports). ${ }^{9-12}$

\section{Excessive reliance on Impact Factor and other metrics}

Google Scholar, a comprehensive database of the latest research papers and references, is used by researchers in different fields because it covers most of the published papers in both medical and non-medical journals. Online editions of journals help researcher-authors to obtain a wider readership. The Directory of Open-Access Journals (DOAJ) has recently changed its policy of covering all open-access journals and now excludes many approved journal from its list.

Scopus is another international abstracting and indexing database that seeks to cover all scientific journals from around the world. However, in some cases, good peerreview journals are excluded owing to some unspecified reasons. Since Scopus did not offer any simple citation-based journal metrics to integrate Source-normalized impact per paper (SNIP) and Scimago Journal Rank (SJR), CiteScore was developed as a metric to address this need. CiteScore is calculated by counting the citations received by a Scopusindexed serial title in a given year from any document published in the preceding three years and dividing that number by the number of documents published during the same three-year period. Such a metric can be used to assess the impact of journals within the same subject field. ${ }^{12,13}$

A journal's impact factor (IF) often has a key role in the journal's evaluation or stature. The factor is calculated based on citations from the journals listed in the multidisciplinary citation database Web of Science, which is derived from the collections that constitute the Science Citation Index and the Social Science Citation Index. Thus, the IF does not represent all published scientific journals in the world. Besides, the citations are limited only to the Web of Science database instead of other possible sources. The Impact Factor is therefore unsuitable for evaluating and ranking journals from different disciplines and is not a valid means for evaluating the work of individuals.

Several studies have criticized the IF. ${ }^{13}$ It has been shown that using Citescore (to evaluate the citation impact of titles 
within the same subject field) and CiteScore percentile (for comparing the citation impact of titles in different fields) would be helpful in identifying other suitable metrics. ${ }^{13}$

\section{Scientific misconduct}

Authors sometimes use, consciously or unconsciously, some previously published text in their manuscripts without attribution, a practice that is referred to as plagiarism and found to be more extensive in manuscripts from nonAnglophone countries. ${ }^{3,4}$ Some fake journals, which are never published, exist only in the form of a website that serves as a channel to accept papers- and fees to publish those papers. Such fake journals or enterprises also exist in Iran. ${ }^{14}$

Journal editors should assess the originality of research, the validity of the methodology, the integrity of the results, and the fairness and balance of the conclusions in a research paper and should also take into account any relevant ethical standards. Astaneh (2015) maintains that different factors such as culture, resources, and politics can influence ethical approaches in different countries. ${ }^{12}$ The culture of many developing countries compels students to add the name of their professors in any research articles: professors believe that students owe that knowledge to them. Rejection of papers written by authors from countries being under sanctions is another unethical practice that confronts researchers in those countries. ${ }^{12}$

Another form of misconduct practised by open-access predatory journals is to charge authors a publication fee and, as though in return, dispense with peer review and other standard editorial processes and safeguards. ${ }^{6}$

\section{Discussion}

The shortcomings of local journal can be summarized as inadequate infrastructure, weak publication ethics, carelessly prepared manuscripts, and amateur staff, editors, and reviewers. All these inadequacies lead to insufficiently rigorous and delayed peer review and delays in publishing. Delays in online publication and problems related to a journal's home page lead to fewer citations and thus to a lower IF. Other problems with journals are duplication, high costs of running the journal's office, lack of value addition, failure to reach other countries in the developing world, and insufficiently motivated reviewers.

Best peer reviewers are not necessarily senior scientists or academics: even the young and those recently graduated can be trained as reviewers. The training courses should be for both editors and reviewers. The need for a training programme in science journalism at the postgraduate level has now been recognized in Iran.

Few countries in the world have such courses as part of a curriculum, and Iran is among them that offer this specialty. The programme, offered since 2009, confers a master's degree in science. ${ }^{15}$

The curriculum forms an important educational programme to promote writing in the field of medical sciences, and those who graduate in this discipline can assist faculty members in improving the editorial and technical content of articles.
As science contributes substantially to the economies of developing countries such as Vietnam, the cost of science education must be put into perspective. ${ }^{16}$ The ways described in an article on how members of the editorial board and peer review policies helped in improving Croatian journals ${ }^{17}$ can also be used for improving science journals from Iran. Recommended procedures can be applied to deal with retracted papers. ${ }^{18}$

A new obstacle to journal publishing in Iran is the extension of political sanctions to encompass scientific publishing. This challenge may, in turn, lead to other new problems, such as the inability to keep to publishing schedules, poor accessibility and coverage, technical problems related to internet access, and severe inflation. Over the last three years, inflation is one of the main reasons for the rising cost of life and of research in Iran. As prices have gone up, the value of the Iranian rial has gone down, which means reasearchers need to spend more to complete their studies. Obviously, reduced financial support to research may limit research quality.

Ten years on from the sanctions, Iranian researchers, authors, and editors (as well as other non-academic individuals) continue to face economic problems. However, at least in some cases these chalenges have created some opportunities in the form of willingness to work harder and to forge alliances, greater sympathy from the rest of the world, and greater frugality. Under these circumstances, the following are some of the recommended measures to improve Iranian journals: developing systems of data sharing, promoting greater coordination among scientific journals, optimizing the process of peer review, utilizing open-access publishing models actively, lowering expenses, and exploring ways to increase revenues. Although lack of novelty in most cases is not realated to the above problems, originality can be fostered by introducing undergraduate students to research early on, imparting training in writing and research methodology at the undergraduate level, and organizing courses, seminars, symposia, workshops, and so on for authors and reviewers. Such events and courses need to be organized regularly to stay in the game and to improve the scientific community in Iran and other developing countries. To train the editorial staff and to enlist reliable and hard-working editors is the need of the hour, and closer collaboration needs to be planned among the countries striving to have high-quality journals.

A few more specific and practical suggestions for managing a journal successfully are as follows: working harder on the content rather than on technical issues (cutting down the time spent on proof-reading and layout, for example), insisting on clear citations and accurate referencing, promoting the journal, publishing on schedule but without sacrificing quality, eliminating hard copies, streamlining work flow, making the journal more attractive to a wider readership, and contacting scientists and authors proactively rather than waiting for them to contact the journal. Finally, journals should make real scientific contributions, consider the authors' need for recognition, set up efficient editorial processes, and learn to detect fraud and plagiarism. 


\section{Acknowledgements}

We would like to thank many members of the academic community for their sincere cooperation. We also acknowledge the financial support from the Shahid Beheshti University of Medical Sciences (grant number 17868).

\section{References}

1 Saberi-Karimian M, Afshari R, Movahhed S, Amiri F, Keykhaee F, Mohajer F, Noormandipour M, Lamsehchi A, Nasiri M, Barkhidarian B, Norouzy A. Different aspects of scientific misconduct among Iranian academic members, European Science Editing, 44(2) 2018, 28-31

2 Bipeta R, Medical editing in India, European Science Editing 41(3) 2015; 66-70.

3 Jawaid SA. Problems of editing a peer-reviewed biomedical journal in a developing country. The Journal of Tehran University Heart Center. 2008; 3: 187-190.

4 Jawaid SA, Jafary MH. Simultaneous submission and duplicate publication: curse and a menace which needs to be checked. Pakistan Journal of Medical Sciences. 2005; 21:245-248.

5 Smith R. A beginning that should lead to complete transparency, British Medical Journal. 1999; 2; 318(7175): 4-5, doi: 10.1136/bmj.318.7175.4

6 Marušić A. Discussing predatory journals - EASE at the 5th World Conference on Research Integrity. European Science Editing. 2017; 43(1): 2 .

7 da Costa M, Oliveira J, Abdulmalik J. Where can early career researchers learn how to peer review a scientific paper? European Science Editing. 2018; 44(1): 4-7.

8 Tokalić R, Marušić A. A peer review card exchange game, European Science Editing. 2018; 44(3): 52-55

9 Morcos A. Publishing in developing countries: problems and solutions. CBE Views. 1999; 21: 198.

10 Jafary MH, Jawaid SA. Online edition of a journal: a great source of manuscripts and potential regional cooperation. Pakistan Journal of Medical Sciences. 2006; 22: 107-109.

11 Jawaid SA. Proceedings of workshop on medical editing and peer review held at NICH and DUHS, Karachi, Pakistan. Pakistan Journal of Medical Sciences. 2008; 24: 637-641.

12 Astaneh B. Ethics: an absolute or conditional issue? European Science Editing. 2015; 41(4): 94-94.

13 Colledge L, James C, Azoulay N, Meester W, Plume A. CiteScore metrics are suitable to address different situations - a case study. European Science Editing. 2017; 43(2): 27-31.

14 Larijani B, Niaz K, Pourabbasi A, Khan F, Spoor J, Abdollahi M. Not only Iranian rise in science marred by fraud: Misconduct is a global problem, Experimental and Clinical Sciences. 2017; 16: 1099-1102, doi: $10.17179 /$ excli2017-263

15 http://www.hamshahrionline.ir/details/45318/Communication/ Journalismcommunicational

16 Vuong QH. The (ir)rational consideration of the cost of science in transition economies, Nature Human Behaviour, 2018; DOI: 10.1038/ s41562-017-0281-4:

17 Utrobičić A, Šimić J, Malički M, Marušić M, Marušić A. Composition of editorial boards and peer review policies of Croatian journals indexed in Web of Science and Scopus. European Science Editing 2014;40(2):31-33.

18 Gilliver S. Recommended procedures for retracting articles: Inadequate and patchily applied? Analysis of a recent article in PLoS ONE examining the fates of retracted articles. Medical Writing 2014;23(2):122-124. DOI: 10.1179/2047480614Z.000000000202 